\title{
X. Regenerative medicine
}

In Figure 67 we noted the three main areas to be addressed in health care: diagnostics, therapeutics and restorative or regenerative medicine The two first steps have been addressed in the two preceding chapters. The goal of regenerative medicine, the third main area of health care, is to repair diseased parts of the body. Some people are born with serious deficiencies. Accidents can lead to serious damage of the body: Some parts are destroyed or seriously injured. The aging of populations in many countries also leads to an increasing demand for regenerative medicine.

One opportunity offered by nanotechnology is that it is possible to manufacture objects with properties similar to those of naturally occurring nanoscale structures. This opens the possibility to repair damage to cells or tissues in a way similar to the way biological systems do it, or to manufacture devices with the ability to correct functional deficiencies in the human body.

\section{Biomaterials}

An important requirement for biomaterials is that they be accepted by the surrounding tissues of the patient and remain biologically inert. Biomaterials were already used almost a half a century ago to replace some limited parts of the body. In the late 1980s, bioactive components with a controlled action on the body of the patient were used in the orthopedic and dental domains. Absorbable materials were also developed at that time. A more contemporary goal in this area is the development of biomaterials with regenerative properties providing self-healing capabilities to skin, bones, cartilage and tissue.

Presently, damaged tissues or organs are often replaced by artificial substitutes implanted in the body (hip replacements, coronary artery bypass grafting, coronary stents...). Nanocoatings can improve the surface properties, durability and biocompatibility of such devices. Nanopolymers are used to coat devices in contact with blood, such as catheters to prevent clot formation. Nanofiber membranes can be covered with antibiotics, painkillers or other specific drugs before being applied to diseased or damaged tissues. 
Regenerative engineering is based on development of absorbable scaffolds with appropriate cells capable of regenerating the damaged or diseased tissue. it is possible to produce regeneration messengers for monitoring the tissue reconstruction. Nanostructures could be used to manufacture temporary, biodegradable materials which do not need to be surgically removed after they have done their job. Within the current decade it is expected that the ability to control the growth of ${ }_{3} \mathrm{D}$ scaffolds will be developed.

Living systems are extremely efficient in many respects. Understanding their function can be of great help in regenerative medicine. Biomimetics is the study of the formation, structure, or function of biologically produced substances for the purpose of creating artificial mechanisms which mimic natural ones. We have already discussed some important natural mechanisms in chapter 5 . The physics and chemistry on which they are based can be adapted to development of artificial devices implanted in the body. An important point is to improve as much as possible the longevity, the biocompatibility and the life time of implanted devices. Bionanomaterials can play a key role in this respect. Bioactive coatings can produce better interfaces between artificial implants and the neighboring tissues. Nanocoating can also help to prevent immune rejection mechanics by building a barrier at the interface between tissues. Smart materials which respond to external stimuli such as temperature, $\mathrm{pH}$, electrical signals... can be manufactured in such a way that they respond to the environment in which they are located.

\section{Cell therapy}

Injured tissues heal themselves more or less rapidly depending on their nature. Healing can be rather fast for blood or epidermal injuries but is much slower for bones or cartilage. Cell therapy offers large opportunities in regenerative medicine.

The communication between cells is based on direct cell-to-cell signaling and on the interaction with diffusible biomolecules. This communication process is essential in healing mechanisms. Indeed, a single cell must know what happens in its environment and behave accordingly. Investigations of natural healing mechanisms and identification of the different cell signals present in the communication between cells can be made using different methods. 
Nanoscience can help to understand the complex communication process between cells. In the future it is expected that nanotechnology can replace part of this communication process with smart nanodevices delivering biomolecules (proteins, genes...) at controlled rates to activate cells to produce the growth factors necessary to initiate the healing mechanism of the tissue. Stem cells have an unparalleled ability to regenerate different kind of tissues and nanotechnology can be used to provide delivery vehicles to bring them to the right location.

\section{Implants}

Implants are often needed to replace important parts of the human body. There are two main issues with implants. The first one is that they can be rejected by the body. The second is that they can be worn out and require replacement. Artificial hips, for example, typically need to be replaced after 10-15 years. Nanocoatings can be designed to improve biocompatibility and increase the mechanical resistance to wear. They can also be employed to prevent adherence of biological material; an important property for synthetic vascular grafts and other artificial materials. Vascular implants and stents are extensively used to treat some cardiovascular diseases. Additional capabilities such as protection from bacterial or fungal infections can be incorporated. Nanomaterials can provide lighter weight implants with longer lifetimes than those of conventional implants.

Another important issue for implants requiring electricity to function is provision of an appropriate energy source. Lithium-ion batteries can have lifetimes of the order of 7-10 years. Induction charging is an interesting possibility to recharge the battery without any surgical interaction in the body of the patient.

For repair of bones and teeth, it is necessary to have a good osseointegration of the implant. This depends very much on the interface between the implant and the surrounding bone. It turns out that the larger the surface of the interface, the faster is the healing process. Titanium, a metal widely used in bone implants, has an improved osseointegration in bone repairs when the surface of the implant is nanostructured. Nanotechnology will play a greater role in the future.

Methods to repair damaged cartilage, a problem for a large number of the aging, are also being developed.,. In vitro cartilage tissue regeneration techniques are being explored. New cells generated in this fashion can be 
transplanted into the body. Other areas such as tracheal, esophageal and bladder regeneration can also benefit from such in vitro techniques.

Neural implants present a particularly difficult problem. Silicon nanowires have the ability to detect a larger number of signals coming from the neurons than do conventional electrodes. Because of their good electrical conductivity, carbon nanotubes are being investigated as replacements for axons. Nanodevices are also being developed to electrically stimulate some specific part of the brain for the treatment of neurodegenerative diseases such as Parkinson's disease.

Vision and hearing are two critical senses and progress is currently made to develop retinal and cochlear implants. Nanotechnology allows development of smaller and more powerful devices to restore vision and hearing.

According to the United Nations (UN) The share of elderly people,defined for this purpose to be people older than 60 years, among the global population has increased from $9.2 \%$ in 1990 to $11.7 \%$ in 2013 . The UN foresees this proportion of the world population to reach $21 \%$ by 2050 . This increase results from a decreasing mortality and a decline of fertility. There are presently about 45 million people in the world suffering from blindness, some since birth, others as a result of accidents or disease. This number is increasing as the population ages.

Some of the diseases leading to blindness or severe visual loss cannot currently be prevented or cured. Retinis pigmentosa, an inherited disorder affecting about 1 million of people in the world is one of these. Artificial vision using prosthetic devices is then the unique current solution of the problem. Artificial vision is based on an electric device implanted in the eye to simulate nerve tissues. Electrical signals containing the visual information are transmitted to the brain creating virtual points of light which can create an image for the patient. The technologies under development can be classified according to the coupling between the electrodes of the device and the tissues (http://www.nidek-intl.com/ visual_prosthesis).

A retinal implant (called also artificial retina) stimulates the retina with electrodes. The retina can be stimulated by three major methods: from the top side (epiretinal implant) by implanting an electrode array on the retinal tissue, by implanting an electrode array under the retina (subretinal implant) or the suprachoroidal transretinal stimulation where a stimulation electrode is placed inside the sclera which is the tissue covering the outermost part of the eye and where the return electrode is located in the intravitreous part of the eye.

This is summarized in figure 82 . 


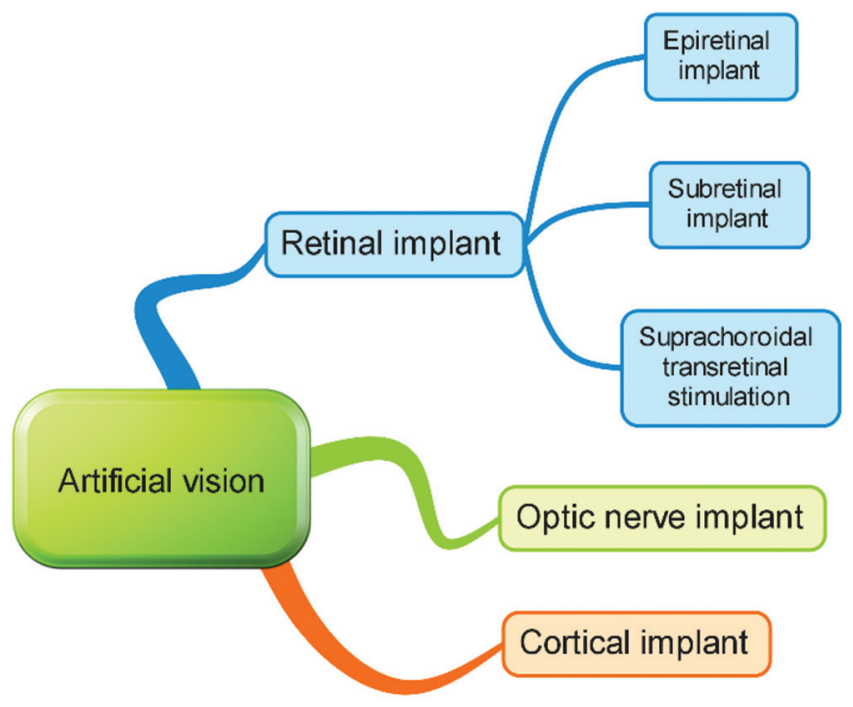

Figure 82. Main routes for enabling artificial vision.

A less advanced technology is a nerve implant where a cuff electrode array is wrapped around the optical nerve. A camera provides an image which is transformed into electric signals and sent to the nerve electrode array to stimulate the optical nerve. Training is necessary and this system requires a lot of software.

Cortical implants are located inside the brain to stimulate directly the visual cortex after transforming the image of a camera into electrical signals. A $5 \times 5$ signal array (25 pixels) provides the ability to read some letters and a $10 \times 10$ array (100 pixels) provides the ability to distinguish some shapes. A minimum resolution of a $32 \times 32$ array (1024 pixels) is necessary to provide a person an image allowing that person to function with some freedom. A retinal prosthesis project with 1500 electrodes has been developed recently in Tübingen, Germany. This is much larger than most of the projects which have less than 100 electrodes.

\section{Dentistry}

The main goal of dentistry is to maintain the health of oral tissues. Nanotechnology can be applied in all areas of dental care, prevention, diagnosis and treatment. 
Periodontal diseases have heavy consequences on the oral cavity: demineralization of the enamel and the dentin, degeneration of the soft tissue and the periodontal ligament, etc. The regeneration of lost tissues is therefore an important issue. Three main strategies are carried out in this respect: bone grafting, bone substitutes and guided tissue regeneration. Nanotechnology can be useful in the last two strategies. Nanohydroxyapatite particles with a size of $18 \mathrm{~nm}$ can be used as bone substitute or in the remineralization of teeth following exposure to carbonated beverages. Nanotubes and carbon nanofibers can also speed up the process of bone regeneration.

Titanium is a metal often used in orthopedics and dentistry thanks to its high fracture resistance, ductility and lightweight. Its disadvantage is that does not enhance cell adhesion and osteoblasts development. Coating the implant with nanofibers or nanostructures can mitigate this problem.

Using nanoparticles with antimicrobial properties allows a decrease in the number of long-term antibiotic treatments with the advantage of reducing the extension of antibiotic resistance. For example, metal nanoparticles such as silver nanoparticles are efficient against Gram negative and anaerobic bacteria. The efficiency depends on several factors such as the concentration of nanoparticles (a too low concentration is inefficient and may induce bacterial resistance), on their sizes (smaller nanoparticles are more efficient than larger sizes particles), on their geometries. Figure 83 summarizes the kind of nanoparticles with antimicrobial activity which can be used in dentistry ${ }^{10}$.

Nanofiber scaffolds can be used to deliver hormones and cells without triggering an immune response in the body when they dissolve. In dentistry, these scaffolds are used for regeneration of oral and dental tissues. (alveolar bone, periodontal ligament, dental pulp, enamel).

Nanotechnology can also be used in drugs delivery systems. The drugs which are encapsulated into nanoparticles are protected during delivery preventing toxic side-effects Nanocoatings, such as those made of silica nanoparticles can be used for the delivery of antibacterial drugs. The delivery can also be triggered by an external signal, such as laser irradiation or using magnetic interactions. Delivery of various agents (antibodies, proteins, cell-targeting drugs) can be made with loaded nanoshells. Nanoshells are nanoparticles with a dielectric core (silica for example) and a thin metal coating (gold for example). Nanoshells can also be used alone because they are sensitive to infrared radiation. Infrared radiation is absorbed by the nanoshell and its temperature increase gives the ability to destroy bacteria, cancer cells etc. 


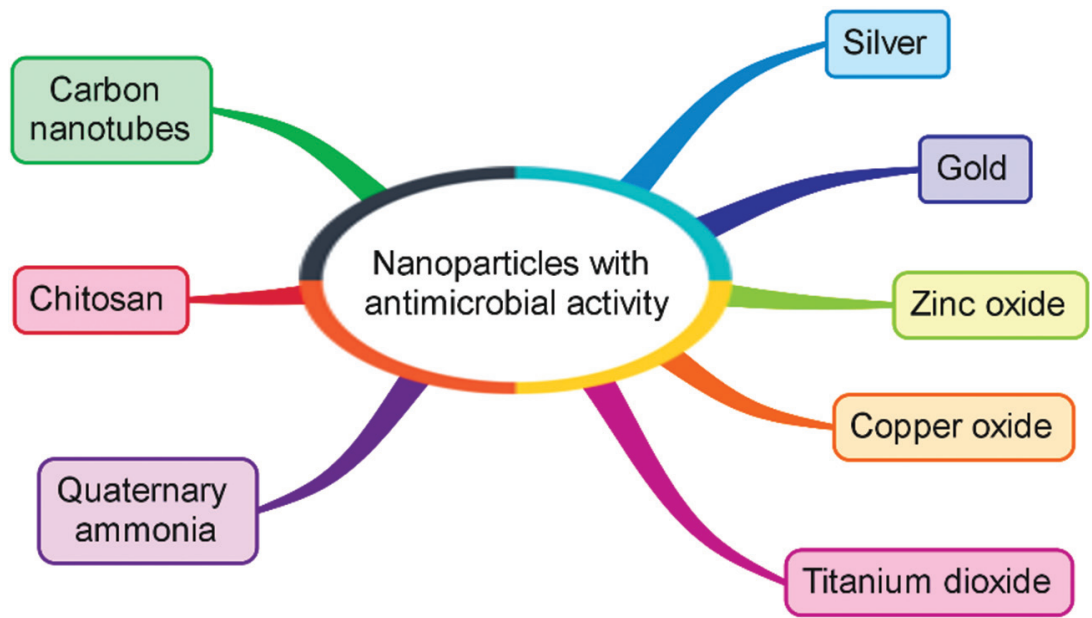

Figure 83. Nanoparticles with antimicrobial activity. Chitosan is a polysaccharide with high biocompatibility and coagulative properties obtained from shrimp and crustacean shells.

Quantum dots nanoparticles of lead sulfide, zinc sulfide, indium sulfide are semiconductors. Quantum dots are also useful in dentistry because they can absorb and reemit light at a definite wavelength which depends on the size of the quantum dot. They can be coated with molecules which have a tendency to attach to cancer cells. The size of the quantum dot can be adjusted so that they absorb and emit in the UV region and can be used in the detection of oral cancers.

\section{Nanosurgery}

The modern trend in surgery is to be minimally invasive lessening patient trauma and promoting prompter patient recovery.

Crystalline or polycrystalline silicon can be used to make surgical blades with cutting edges between 5 and $500 \mathrm{~nm}$. This is useful in eye surgery.

Nanoneedles with a diameter between 200 and $300 \mathrm{~nm}$ can penetrate into living cells without harming them. They allow performance of cell surgery but can also be used to deliver specific drugs to the cell.

Biological molecules inside a cell can be manipulated with nanotweezers with a thickness below $50 \mathrm{~nm}$. This opens the way to perform DNA manipulations and perform physical measurements.

Femtosecond laser surgery is a technique with a great potential in this regard. It uses a high intensity light beam in the near infrared region (740 and 
$800 \mathrm{~nm}$ wavelength). It has the ability to operate on a volume smaller than a femtoliter $\left(10^{-15}\right.$ liters). With this technique, a cut size of $110 \mathrm{~nm}$ has been achieved allowing chromosome dissection within a single living cell. Future applications of this technique in eye surgery, neurosurgery, gene therapy, etc. will have great impact.

\section{Summary}

Some people are born with severe medical problems. Others contract debilitating diseases later in life. Some people get seriously injured in accidents. The population is ageing. For all these reasons there is an increasing demand for regenerative medicine. Biomaterials and implants are one route to regenerative medicine. These areas have been developed for a long time but nanotechnology can support the maintenance of healthy tissue and progress in regenerative medicine, opening possibilities that have not previously existed. Cell therapy offers a powerful technique for self-healing of biological materials such as bones, tissues. Nanosurgery is developing techniques to work at the cell level with the possibility of modifying genes. 